\title{
AN ILL POSED PROBLEM FOR A HYPERBOLIC EQUATION NEAR A CORNER ${ }^{1}$
}

\author{
BY STANLEY OSHER ${ }^{2}$ \\ Communicated by Eugene Isaacson, January 23, 1973
}

The purpose of this note is to give a simple example of an ill posed problem for a hyperbolic equation to be solved in a region whose boundary has a corner. In [2] we gave necessary and sufficient conditions for existence, uniqueness, and the validity of certain energy estimates for the solutions of a general class of these problems. Analogous conditions for problems in regions with smooth boundaries were obtained by Kreiss [1]. Our example below is somewhat unusual in that bounded $C^{\infty}$ initial data lead to a solution which is exponentially unbounded at the corner for any positive time.

Consider the equation

$$
\left(\begin{array}{l}
u \\
v
\end{array}\right)_{t}=\left(\begin{array}{rr}
-1 & 0 \\
0 & 1
\end{array}\right)\left(\begin{array}{l}
u \\
v
\end{array}\right)_{x}+\left(\begin{array}{rr}
1 & 0 \\
0 & -1
\end{array}\right)\left(\begin{array}{l}
u \\
v
\end{array}\right)_{y}
$$

to be solved for the complex valued functions $u$ and $v$ in the region $0<x, y, t$ with initial conditions

$$
u(x, y, 0)=\Phi(x, y), \quad v(x, y, 0)=\psi(x, y),
$$

and boundary conditions

$$
\text { (a) } u(0, y, t)=a v(0, y, t), \quad \text { (b) } v(x, 0, t)=b u(x, 0, t) .
$$

$a$ and $b$ are complex numbers.

We have the following:

THEOREM. The above problem is well posed, i.e. generates a strongly continuous semigroup for $t>0$ on $L_{2}$, if and only if $|a b| \leqq 1$.

We note here that by the results in [1], the half space problem (1), (2) to be solved for $0<x, t ;-\infty<y<\infty$ is well posed for any boundary condition (3)(a), as is the half space problem for $0<y, t ;-\infty<x<\infty$ for any boundary condition (3)(b).

AMS (MOS) subject classifications (1970). Primary 35L50, 35L30; Secondary 78A45.

Key words and phrases. Hyperbolic equations, initial boundary conditions, well posedness, energy estimates.

${ }^{1}$ Research supported under N.S.F. Grant No. GP29-273.

${ }^{2}$ Fellow of the Alfred P. Sloan Foundation. 
Proof. If $|a b|>1$, consider the functions

$$
\text { (4) } \quad u(x, y, t)=a(a b)^{(t-x) /[2(x+y)]}, \quad v(x, y, t)=(a b)^{(t+x) /[2(x+y)]} \text {, }
$$

where the same argument for $a b$ is chosen in both expressions. This pair of functions satisfies the conditions of (1) and (3) with initial data which are bounded and $C^{\infty}$ for $x, y>0$. The initial data are then multiplied by the factor $(a b)^{t /[2(x+y)]}$ which is exponentially unbounded, as is the solution, when $x+y \rightarrow 0$ for any positive $t$. The solution is well behaved for finite $t$ away from $x=y=0$.

If $|a b| \leqq 1$, we choose two positive numbers $c_{1}, c_{2}$ such that $c_{1}|a|^{2} \leqq c_{2}$ and $c_{2}|b|^{2} \leqq c_{1}$. We then have, using (1) and (3):

$$
\frac{d}{d t} \int_{0}^{\infty} \int_{0}^{\infty}\left[c_{1}|u|^{2}+c_{2}|v|^{2}\right] d x d y \leqq 0 .
$$

$L_{2}$ well posedness is immediate.

\section{REFERENCES}

1. H.-O. Kreiss, Initial boundary value problems for hyperbolic systems, Comm. Pure Appl. Math. 3 (1970), 277-298.

2. S. Osher, Initial-boundary value problems for hyperbolic systems in regions with corners. I, Trans. Amer. Math. Soc. 176 (1973), 141-165.

Department of Mathematics, State University of New York, Stony Brook, New YORK 11790 